\title{
Solvent Vapor Annealing Guides Molecules to Form a Desired Stacking Mode According to the Characteristics of Molecular Structure
}

\author{
Chunxiu Guo, Qijian Zhang, Hua Li*, Jianmei Lu*
}

College of Chemistry, Chemical Engineering and Materials Science, Collaborative Innovation Center of Suzhou Nano Science and Technology, Soochow University, Suzhou 215123 (P. R. China), E-mail: lihuaw@suda.edu.cn, lujm@suda.edu.cn

\section{Experimental Section}

\subsection{Materials}

6-bromooxindole, 6-bromoisatin, 1-bromo-2-[2-(2-methoxyethoxy)ethoxy]ethane, potassium carbonate, $\mathrm{Pd}_{2}(\mathrm{dba})_{3}, \mathrm{P}(\mathrm{o}-\mathrm{tyl})_{3}, \mathrm{~K}_{3} \mathrm{PO}_{4}$, 5-cyanothiophene-2-boronic acid and all the solvents were purchased from Shanghai Chemical Reagent Co. Ltd. All chemicals were used as received without further purification.

\subsection{Instrumentation and characterizations}

Thermo-gravimetric analysis (TGA) was conducted on a TA Instruments Dynamic TGA 2950 at a heating rate of $10{ }^{\circ} \mathrm{C} \cdot \mathrm{min}^{-1}$ and under an $\mathrm{N}_{2}$ flow rate of $50 \mathrm{~mL} \cdot \mathrm{min}^{-1}$. Differential scanning calorimetry (DSC) was tested of $\mathrm{N}_{2}$ atmosphere, the test range of 
the two heating process is $25 \sim 220^{\circ} \mathrm{C}$, and the heating rate is $10{ }^{\circ} \mathrm{C} \cdot \mathrm{min}^{-1}$; the test range of the cooling process is $220 \sim 25^{\circ} \mathrm{C}$, and the cooling rate is $20^{\circ} \mathrm{C} \cdot \mathrm{min}^{-1}$.Ultravioletvisible (UV-Vis) absorption spectra were obtained with the use of a Shimadzu UV3600 spectrometer. Cyclic voltammetry was performed on a CorrTest CS electrochemical station at room temperature. X-ray diffraction (XRD) pattern was taken on a Thermo Fisher Scientific EQUINOX 5000 X-ray diffraction meter. AFM images were collected on Bruker Icon AFM platform in taping model then analyzed and exported by NanoScope Analysis software form the same corporation. I-V spectra were taken from the Tektronix Keithley 4200 semiconductor parameter analyzer under ambient condition. ${ }^{1} \mathrm{H}$ NMR spectra were measured on an Inova $400 \mathrm{MHz}$ FT-NMR workstation.

\subsection{Synthetic procedures}
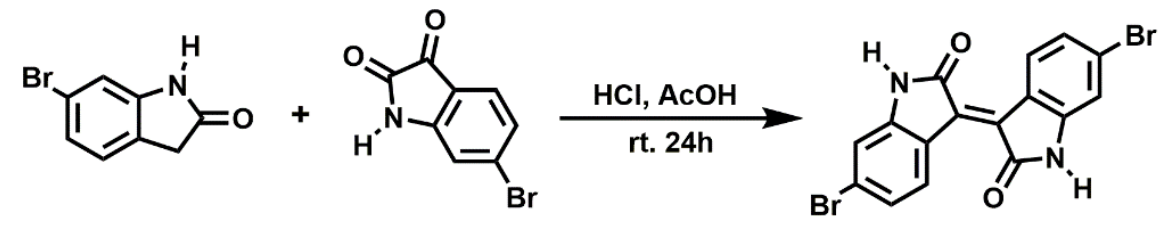

1

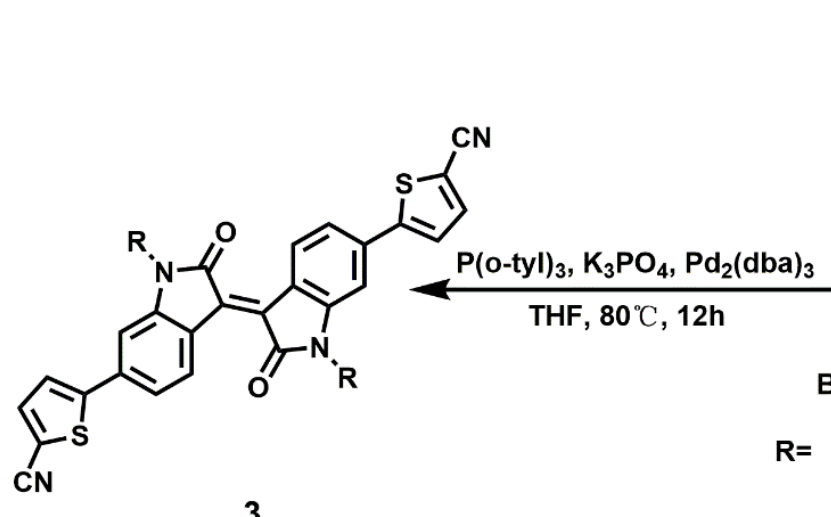

3

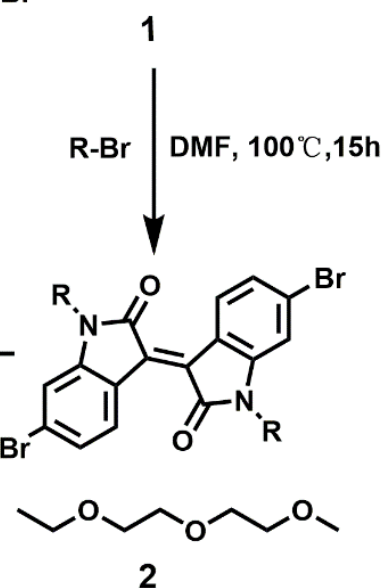

Scheme S1. Synthesis scheme of small molecule TEG-ID(TPCN)2.

Synthesis of 6,6'-dibromoisoindigo (1). 6-Bromooxindole (1.71 g, $8 \mathrm{mmol}$ ) and 6- 
bromoisatin $(1.81 \mathrm{~g}, 8 \mathrm{mmol})$ were diluted in $\mathrm{AcOH}(25 \mathrm{~mL})$. Then the conc. $\mathrm{HCl}$ solution $(0.6 \mathrm{~mL})$ was added to the mixture and the reaction mixture was kept under stirring and reflux for $24 \mathrm{~h}$. After cooling, the mixture was filtered. The solid was successively washed with water, ethyl alcohol and ethyl acetate. After drying under vacuum, 6,6'-dibromoisoindigo was obtained as a brown powder (90\%).

${ }^{1} \mathbf{H}$ NMR (400 MHz, DMSO-d $\left.{ }_{6}, \mathrm{ppm}\right): \delta 11.11(\mathrm{~s}, 2 \mathrm{H}), 8.99(\mathrm{~d}, \mathrm{~J}=8.6 \mathrm{~Hz}, 2 \mathrm{H}), 7.19$ $(\mathrm{dd}, \mathrm{J}=8.6,2.1 \mathrm{~Hz}, 2 \mathrm{H}), 6.99(\mathrm{~d}, \mathrm{~J}=2.0 \mathrm{~Hz}, 2 \mathrm{H})$.

Synthesis of 6,6'-dibromo-N,N'-TEG-isoindigo (2). Under a nitrogen atmosphere, the compound 6,6'-dibromoisoindigo $(2.11 \mathrm{~g}, 5 \mathrm{mmol})$ and potassium carbonate $(2.76$ g, $20 \mathrm{mmol})$ were dissolved in DMF $(60 \mathrm{~mL})$ and the reaction mixture was stirred for about $30 \mathrm{~min}$. Then 1-bromo-2-[2-(2-methoxyethoxy)ethoxy]ethane (2.73 g, $12 \mathrm{mmol})$ was injected, and then the mixture was stirred for $15 \mathrm{~h}$ at $100^{\circ} \mathrm{C}$. After the reaction, the mixture was cooled, poured into water and extracted with EA. The organic layers were washed with brine and water, and then dried with anhydrous $\mathrm{MgSO}_{4}$. Afterwards, the solvent was removed by rotational evaporation, the residue was purified by silica chromatography (eluent EA/PE $=1 / 1(\mathrm{v} / \mathrm{v})$ ), and 6,6'-dibromo-N,N'-TEG-isoindigo was obtained as a red powder (50\%).

${ }^{1}$ H NMR (400 MHz, DMSO- $\left.d_{6}, \mathrm{ppm}\right): \delta 9.01(\mathrm{~d}, J=8.6 \mathrm{~Hz}, 2 \mathrm{H}), 7.41(\mathrm{~d}, J=1.9 \mathrm{~Hz}$, 2H), $7.24(\mathrm{dd}, J=8.6,1.8 \mathrm{~Hz}, 2 \mathrm{H}), 3.97(\mathrm{t}, J=5.3 \mathrm{~Hz}, 4 \mathrm{H}), 3.66(\mathrm{t}, J=5.3 \mathrm{~Hz}, 4 \mathrm{H})$, $3.52(\mathrm{dd}, J=6.0,3.4 \mathrm{~Hz}, 4 \mathrm{H}), 3.45-3.40(\mathrm{~m}, 8 \mathrm{H}), 3.31(\mathrm{t}, J=3.1 \mathrm{~Hz}, 4 \mathrm{H}), 3.16(\mathrm{~s}$, $6 \mathrm{H})$.

(E)-6,6'-Bis(benzo[b]thiophen-2-yl)-1,1'-TEG-[3,3'-biindolinylidene]-2,2'-dione 
(3). The compound 6,6'-dibromo-N,N'-TEG-isoindigo (0.80 g, $1.12 \mathrm{mmol}), \mathrm{Pd}_{2}(\mathrm{dba})_{3}$ (150 mg), $\mathrm{P}(\mathrm{o}-\mathrm{tyl})_{3}(100 \mathrm{mg})$ and $\mathrm{K}_{3} \mathrm{PO}_{4}(2.37 \mathrm{~g}, 11.2 \mathrm{mmol})$ were dissolved in THF (50 $\mathrm{mL}$ ). The mixture was heated to $45^{\circ} \mathrm{C}$ under a nitrogen atmosphere for $0.5 \mathrm{~h}$. 5 cyanothiophene-2-boronic acid $(0.12 \mathrm{~g}, 7.84 \mathrm{mmol})$ was diluted in THF $(10 \mathrm{~mL})$ and the solution was transferred to the mixture through a septum. Then, the mixture was heated to $80^{\circ} \mathrm{C}$ and stirred for $12 \mathrm{~h}$. After the reaction, the cooled solution was extracted with EA. The organic portions were combined and washed with brine and water, and then dried with anhydrous $\mathrm{MgSO}_{4}$. After removing the solvent by rotational evaporation, the residue was purified by silica chromatography, eluting with $\left(\mathrm{CH}_{2} \mathrm{Cl}_{2} / \mathrm{MeOH}=20 / 1, \mathrm{v} / \mathrm{v}\right)$. Finally product 3 was obtained as a black solid $(40 \%)$.

${ }^{1}$ H NMR (400 MHz, DMSO- $\left.d_{6}, \mathrm{ppm}\right): \delta 9.07$ (d, $\left.J=8.4 \mathrm{~Hz}, 2 \mathrm{H}\right), 7.99(\mathrm{~d}, J=4.0 \mathrm{~Hz}$, 2H), $7.82(\mathrm{~d}, J=4.0 \mathrm{~Hz}, 2 \mathrm{H}), 7.41(\mathrm{~d}, J=1.8 \mathrm{~Hz}, 2 \mathrm{H}), 7.32(\mathrm{dd}, J=8.3,1.7 \mathrm{~Hz}, 2 \mathrm{H})$, $4.02(\mathrm{~d}, J=5.8 \mathrm{~Hz}, 4 \mathrm{H}), 3.71(\mathrm{t}, J=5.5 \mathrm{~Hz}, 4 \mathrm{H}), 3.55(\mathrm{dd}, J=5.7,3.6 \mathrm{~Hz}, 4 \mathrm{H}), 3.45$ $(\mathrm{dd}, J=5.8,3.5 \mathrm{~Hz}, 4 \mathrm{H}), 3.39-3.37(\mathrm{~m}, 4 \mathrm{H}), 3.28-3.25(\mathrm{~m}, 4 \mathrm{H}), 3.13(\mathrm{~s}, 6 \mathrm{H})$.

${ }^{13}$ C NMR (101 MHz, Chloroform-d): $\delta$ 167.96, 150.95, 146.12, 138.44, 135.59, 132.43, $130.51,124.38,122.35,119.93,114.11,109.14,106.52,71.85,70.80,70.69,70.57$, $69.31,59.01,40.65$. 


\section{Figures}

(a)

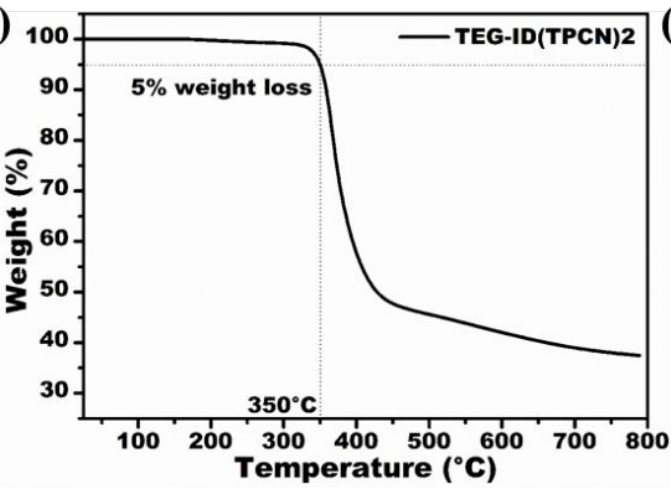

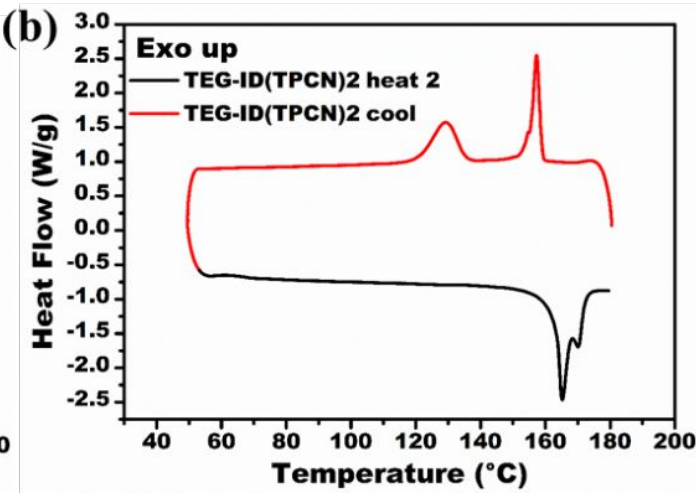

Figure S1. (a) TGA thermogram of the small molecule TEG-ID(TPCN)2 and (b)

DSC graphics of the small molecule TEG-ID(TPCN)2.
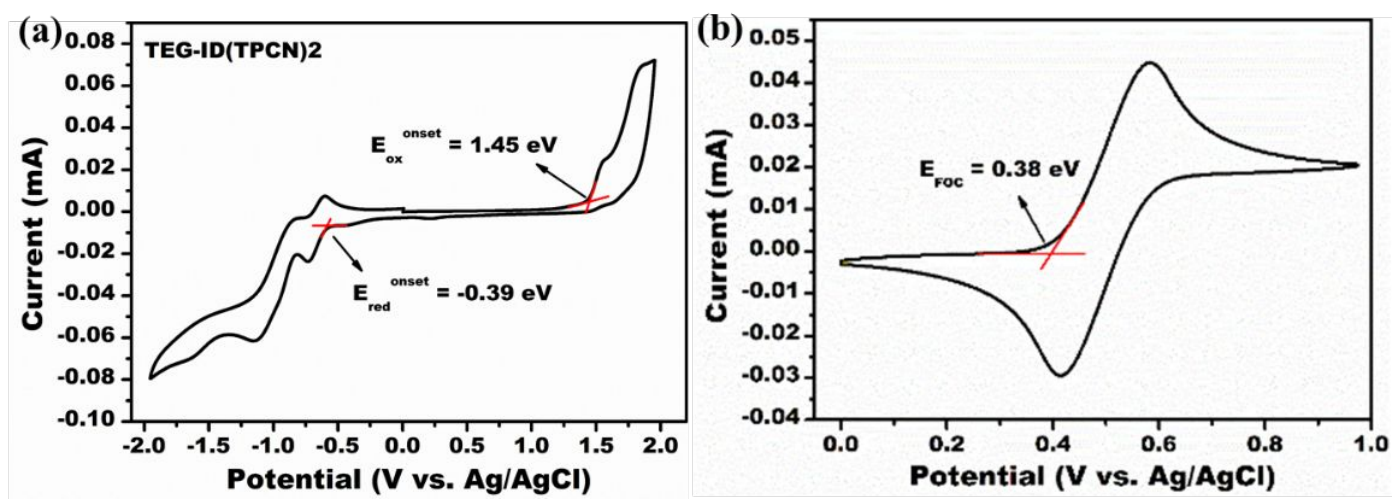

Figure S2. (a) Cyclic voltammetry curves of small molecule TEG-ID(TPCN)2 and

(b) Cyclic voltammetry curves of the ferrocene. 
(a)

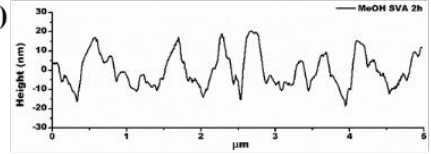

(d)
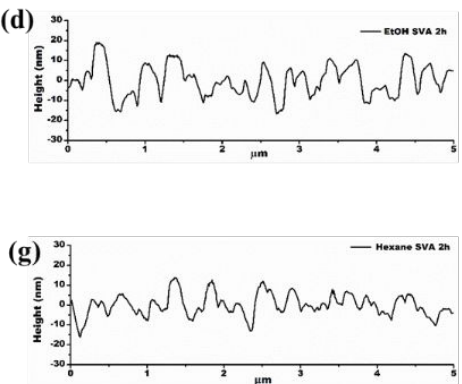

(j)

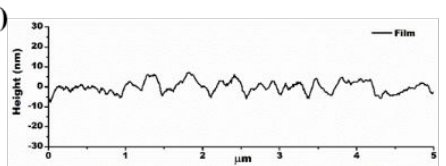

(b)

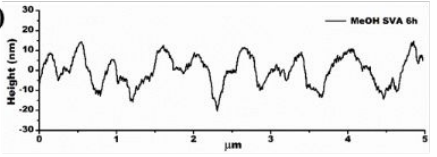

(e)

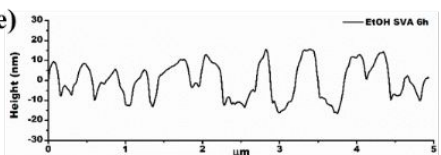

(h)

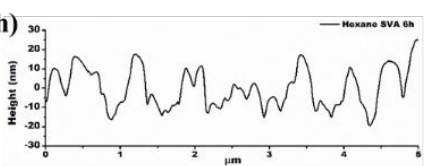

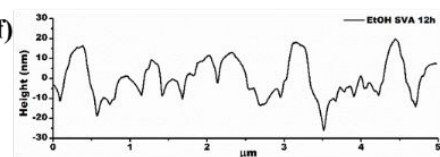

(c)

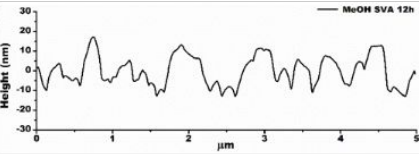

(i)

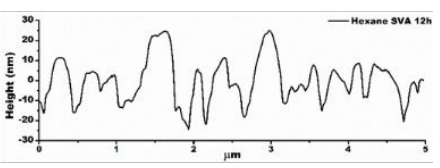

Figure S3. Typical cross-section profile of AFM topographic image of TEGID(TPCN)2 (a-c) MeOH-annealed 2, 6, 12 h, (d-f) EtOH-annealed 2, 6, 12 h, (g-i) Hexane-annealed 2, 6, 12 h, (j)un-annealed film.
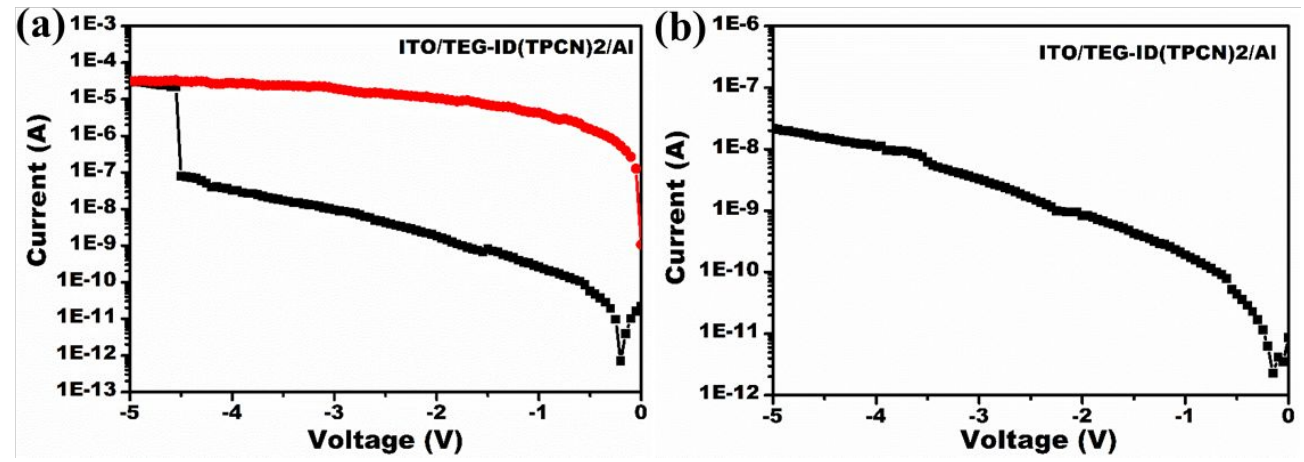

Figure S4. (a) I-V curves for devices fabricated from TEG-ID(TPCN)2 films of ITO/small molecule/ Al structured devices. 

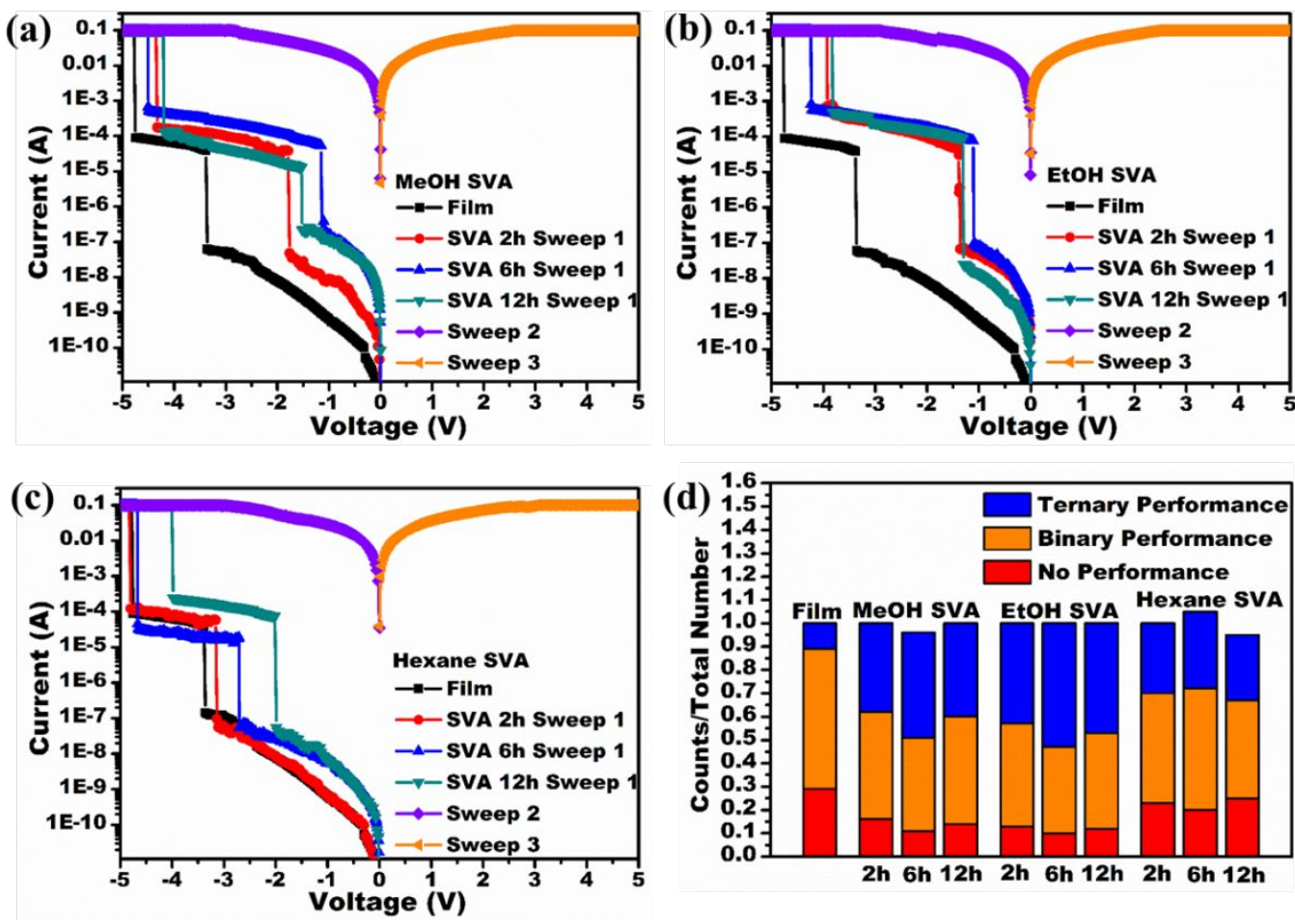

Figure S5. (a) I-V curves for devices fabricated from TEG-ID(TPCN)2 films without and with annealed by $\mathrm{MeOH}, \mathrm{EtOH}$, Hexane. (b) The reproducibility for devices.
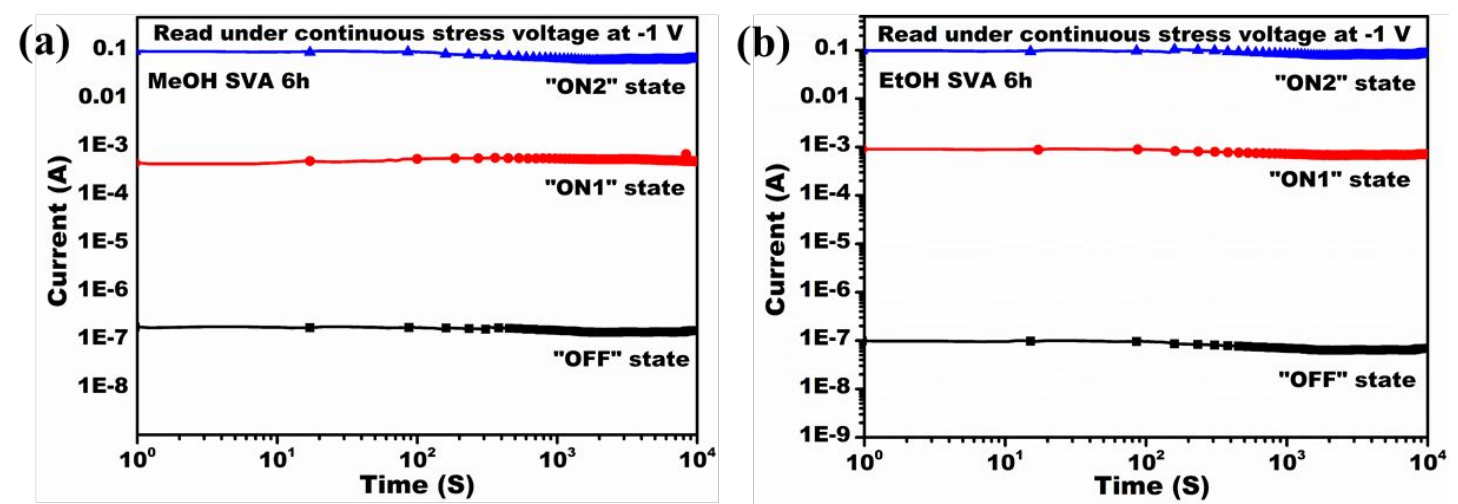

Figure S6. Stability test of the memory devices in all states under a constant reading voltage at $-1 \mathrm{~V}$ (a) through MeOH SVA 6 h, (b) through EtOH SVA $6 \mathrm{~h}$. 

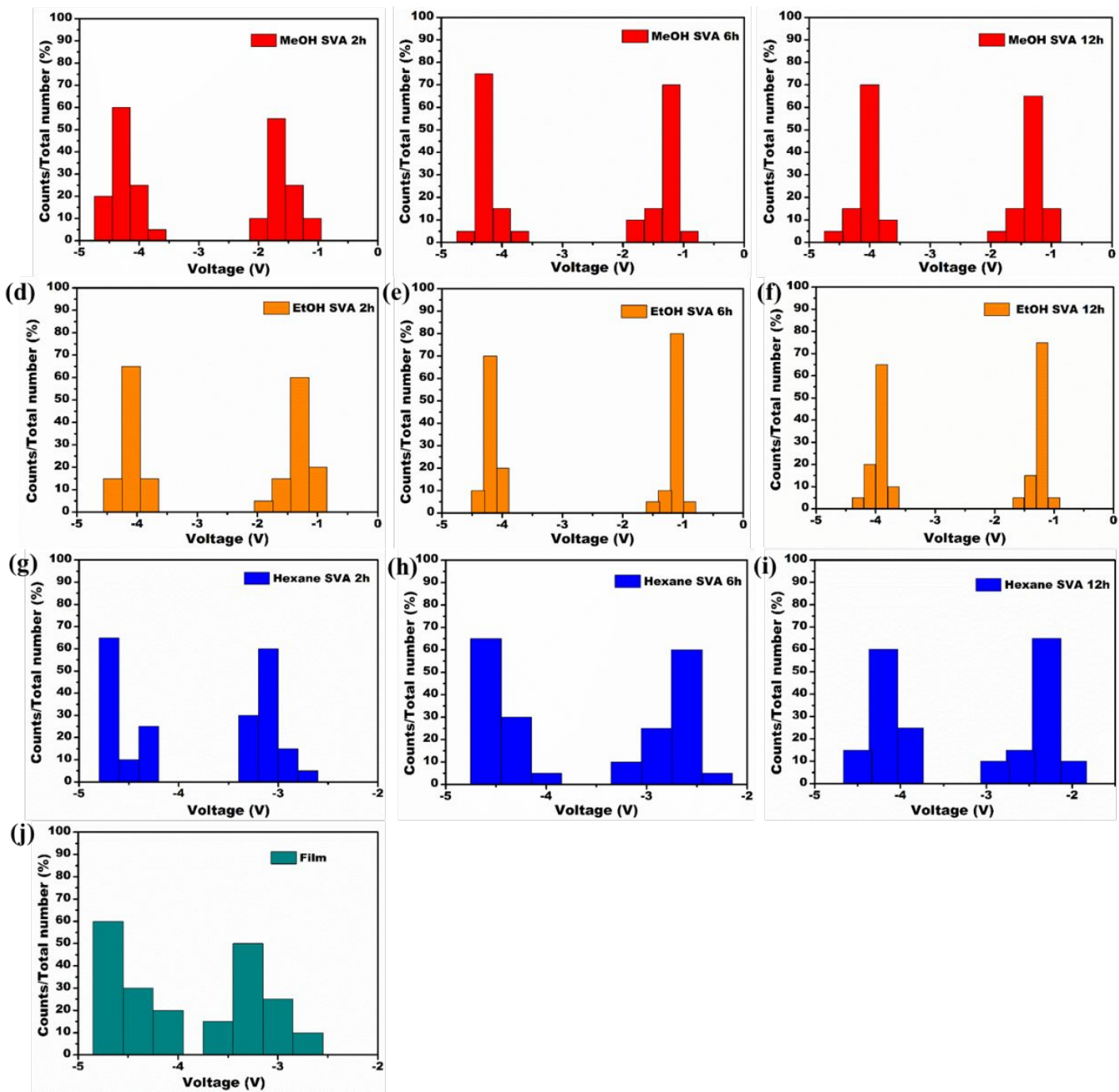

Figure S7. The distribution of switching threshold voltages $\left(\mathrm{V}_{\mathrm{th}} \mathrm{s}\right)$ of TEG-

ID(TPCN)2-based memory devices (a-c) MeOH-annealed 2, 6, 12 h, (d-f) EtOHannealed 2, 6, $12 \mathrm{~h}$, (g-i) Hexane-annealed 2, 6, $12 \mathrm{~h}$, (j)un-annealed film. 


\section{Table}

Table S1. Optical and Electrochemical properties of TEG-ID(TPCN)2.

\begin{tabular}{|c|c|c|c|c|c|c|c|}
\hline molecule & $\begin{array}{l}\lambda_{\text {onset }} \\
(\mathrm{nm})\end{array}$ & $\begin{array}{l}E_{\mathrm{g}} \mathrm{a} \\
(\mathrm{eV})\end{array}$ & $\begin{array}{l}E_{\text {onset }} \\
(\mathrm{eV})\end{array}$ & $\begin{array}{l}\text { b HOMO } \\
\text { c }(e V)\end{array}$ & $\begin{array}{l}\text { LUMO } \\
\text { d (eV) }\end{array}$ & $\begin{array}{l}\boldsymbol{\Phi}_{\mathrm{ITO}^{-}} \\
\mathbf{E}_{\text {HOMO }} \\
(\mathrm{eV})\end{array}$ & $\begin{array}{l}\mathbf{E}_{\mathrm{LUMO}^{-}} \\
\boldsymbol{\Phi}_{\mathrm{AI}} \\
(\mathrm{eV})\end{array}$ \\
\hline TEG-ID(TPCN)2 & 647 & 1.92 & 1.45 & -5.87 & -3.83 & 1.07 & 0.45 \\
\hline \multicolumn{8}{|c|}{ a The data were calculated by the following equation: bandgap $=1240 / \lambda_{\text {onset }}$ of the three } \\
\hline \multicolumn{8}{|c|}{ synthesized molecular films. ${ }^{b} \mathrm{Vs} \mathrm{Ag} / \mathrm{AgCl} .{ }^{c} \mathrm{Calculated}$ from cyclic voltammetry. ${ }^{d}$} \\
\hline \multicolumn{8}{|c|}{ Calculated from the HOMO energy level and optical bandgap. } \\
\hline
\end{tabular}

Table S2. XRD data results of TEG-ID(TPCN)2.

\begin{tabular}{ccc}
\hline & $2 \theta(\mathrm{deg})$ & $\mathrm{d}(\AA)$ \\
\hline TEG- & 6.29 & 14.04 \\
ID(TPCN)2 & 12.60 & 7.02 \\
\hline & 6.81 & 12.97
\end{tabular}

MeOH-2h

13.58 6.52

6.80

12.99

МeOH-6h

13.61 $\quad 6.50$

MeOH-12h

6.84 12.91 


\begin{tabular}{|c|c|c|}
\hline & 13.58 & 6.52 \\
\hline \multirow{3}{*}{ EtOH-2h } & 6.84 & 12.91 \\
\hline & & \\
\hline & 13.63 & 6.49 \\
\hline \multirow{3}{*}{ EtOH-6h } & 6.85 & 12.89 \\
\hline & & \\
\hline & 13.63 & 6.49 \\
\hline \multirow{3}{*}{ EtOH-12h } & 6.83 & 12.93 \\
\hline & & \\
\hline & 13.62 & 6.50 \\
\hline \multirow{3}{*}{ Hexane-2h } & 6.74 & 13.10 \\
\hline & & \\
\hline & 13.57 & 6.39 \\
\hline \multirow{3}{*}{ Hexane-6h } & 6.75 & 13.08 \\
\hline & & \\
\hline & 13.58 & 6.52 \\
\hline \multirow{3}{*}{ Hexane-12h } & 6.74 & 13.10 \\
\hline & & \\
\hline & 13.58 & 6.52 \\
\hline
\end{tabular}

Table S3. Percentage of different storage states of ITO/small molecule/LiF/Al structured devices

\begin{tabular}{ccc}
\hline Ternary & Binary & No \\
performance & performance & performance \\
\hline
\end{tabular}




\begin{tabular}{cccc}
\hline TEG-ID(TPCN)2 & $11 \%$ & $60 \%$ & $29 \%$ \\
\hline MeOH-2h & $38 \%$ & $46 \%$ & $16 \%$ \\
MeOH-6h & $45 \%$ & $40 \%$ & $11 \%$ \\
MeOH-12h & $40 \%$ & $46 \%$ & $14 \%$ \\
\hline EtOH-2h & $43 \%$ & $44 \%$ & $13 \%$ \\
EtOH-6h & $53 \%$ & $37 \%$ & $10 \%$ \\
\hline EtOH-12h & $47 \%$ & $41 \%$ & $12 \%$ \\
\hline Hexane-2h & $30 \%$ & $47 \%$ & $23 \%$ \\
\hline Hexane-6h & $33 \%$ & $52 \%$ & $20 \%$ \\
\hline & & $42 \%$ & \\
\hline
\end{tabular}




\section{$4{ }^{1} \mathrm{H}$ NMR spectra}

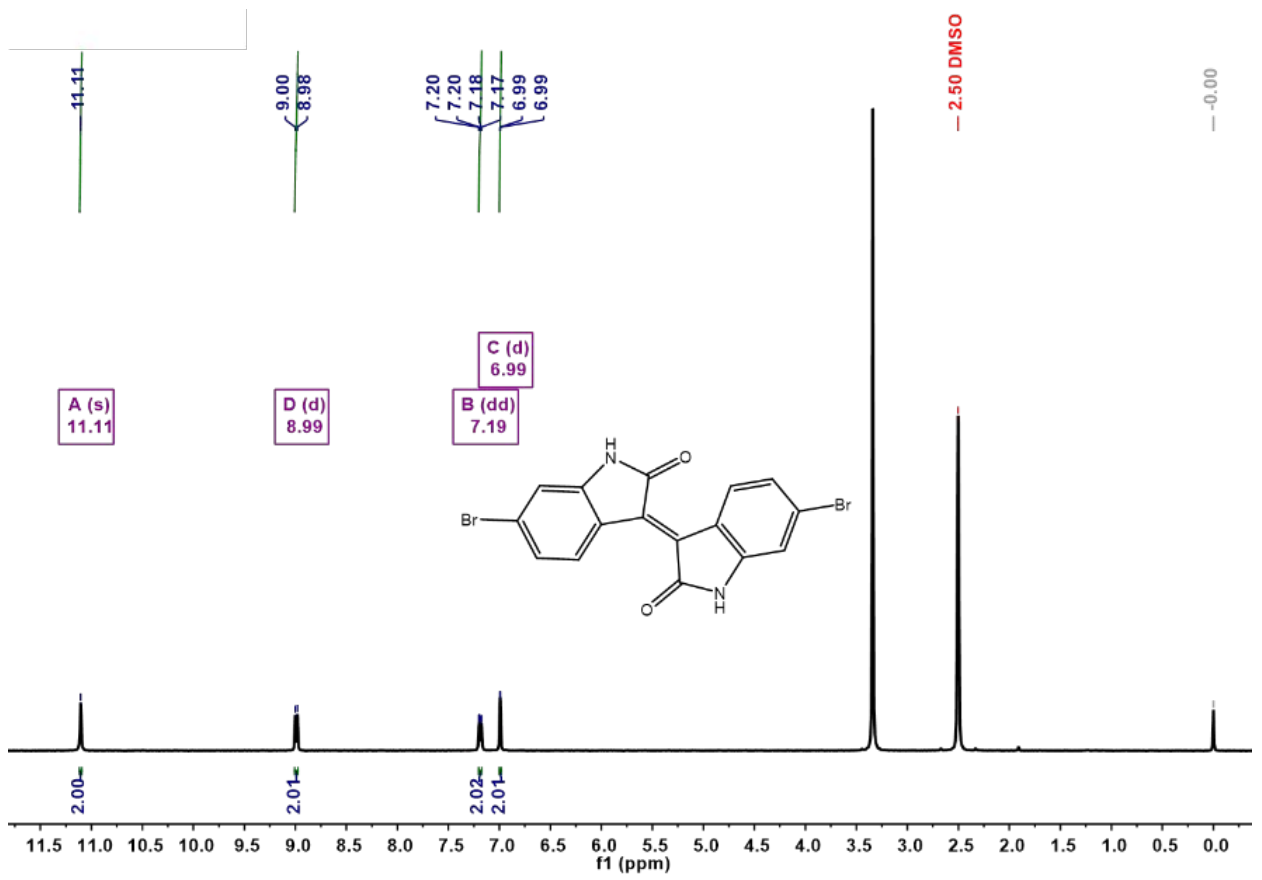

Figure S8. ${ }^{1} \mathrm{H}$ NMR spectrum of 6,6 '-dibromoisoindigo in DMSO- $d_{6}$ with TMS as internal standard.

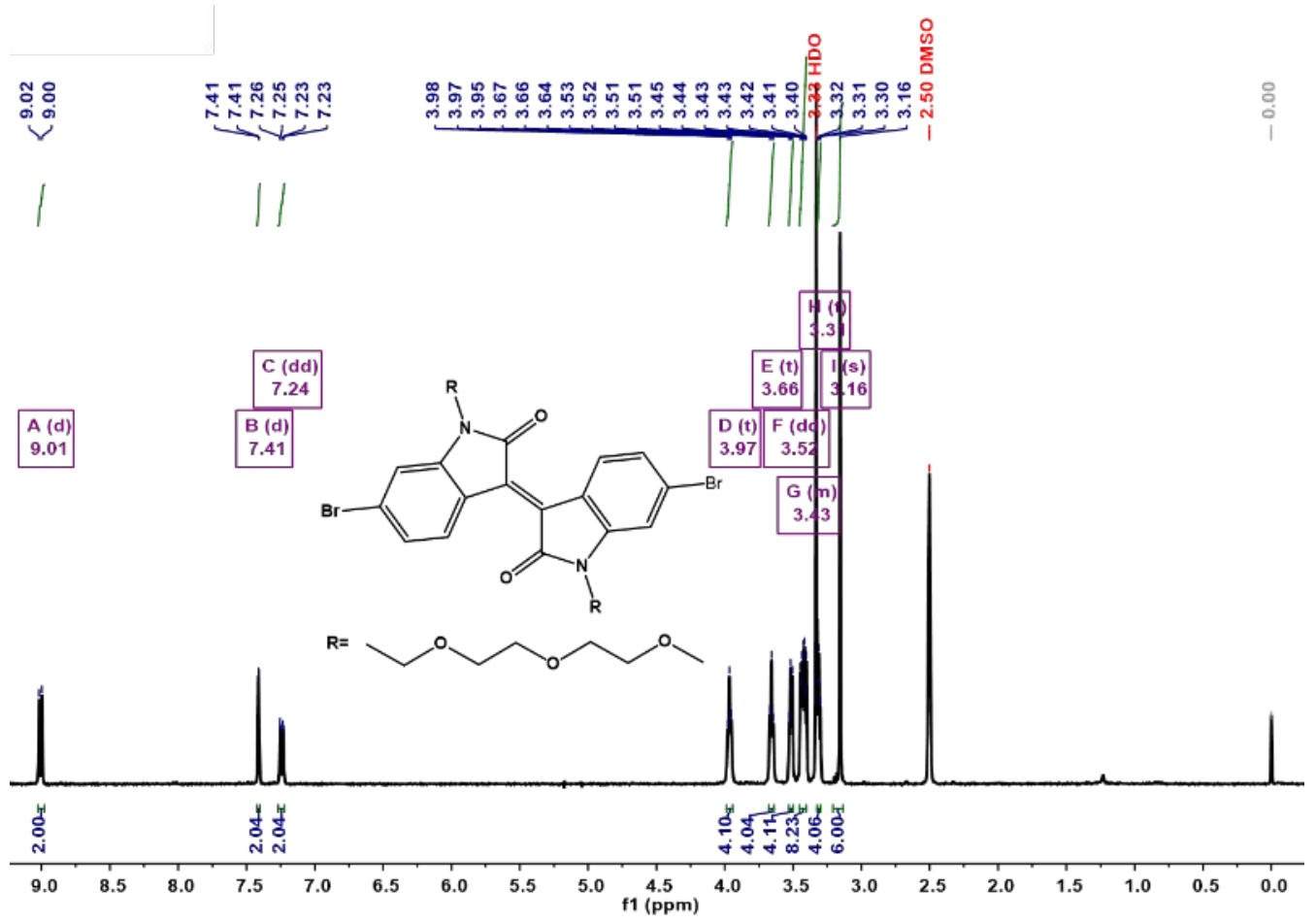

Figure S9. ${ }^{1} \mathrm{H}$ NMR spectrum of 6,6'-dibromo-N,N'-TEG-isoindigo in DMSO- $d_{6}$ with TMS as internal standard. 


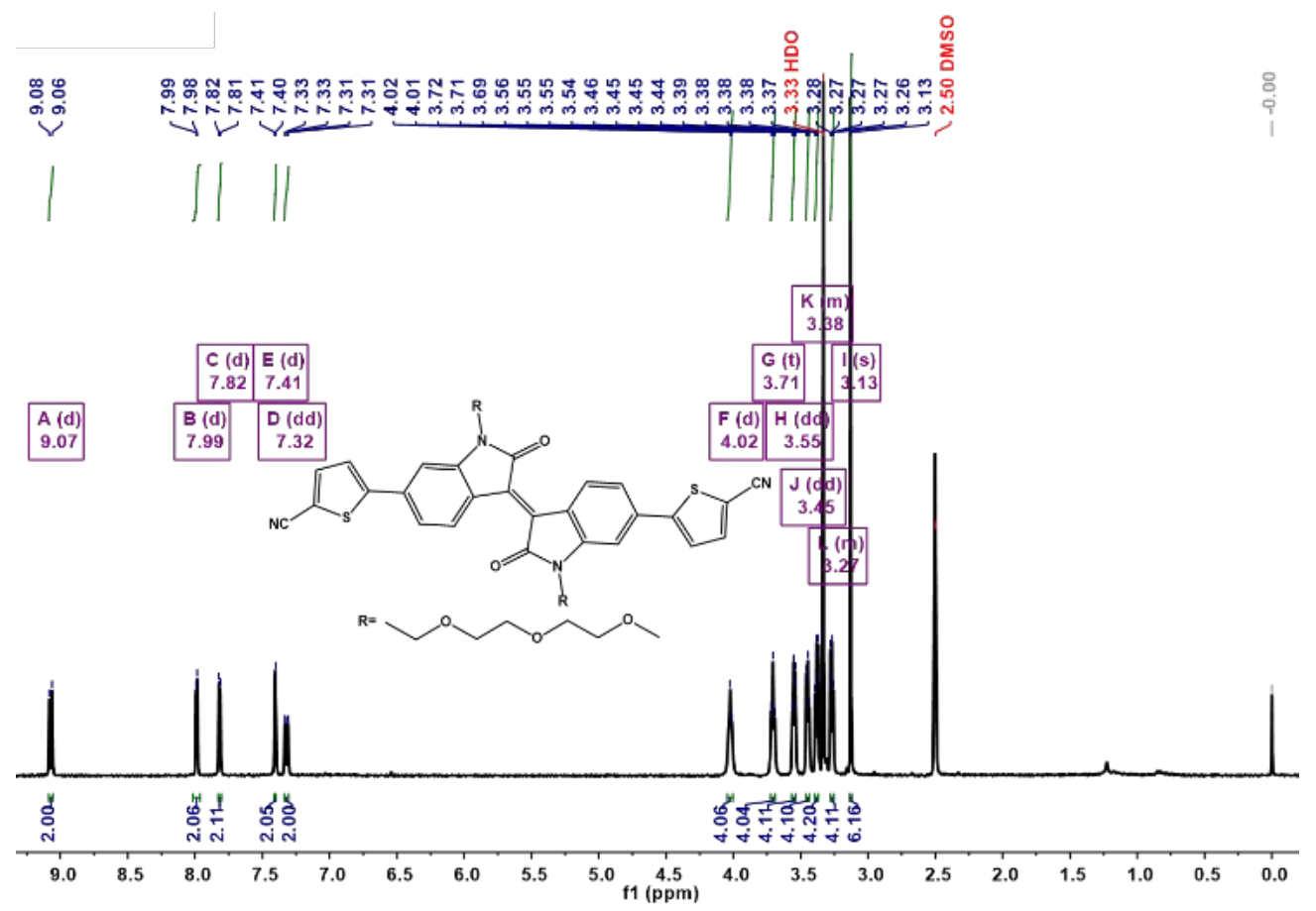

Figure S10. ${ }^{1} \mathrm{H}$ NMR spectrum of TEG-ID(TPCN)2 in DMSO- $d_{6}$ with TMS as internal standard.

\section{$5{ }^{13} \mathrm{C}$ NMR spectra}

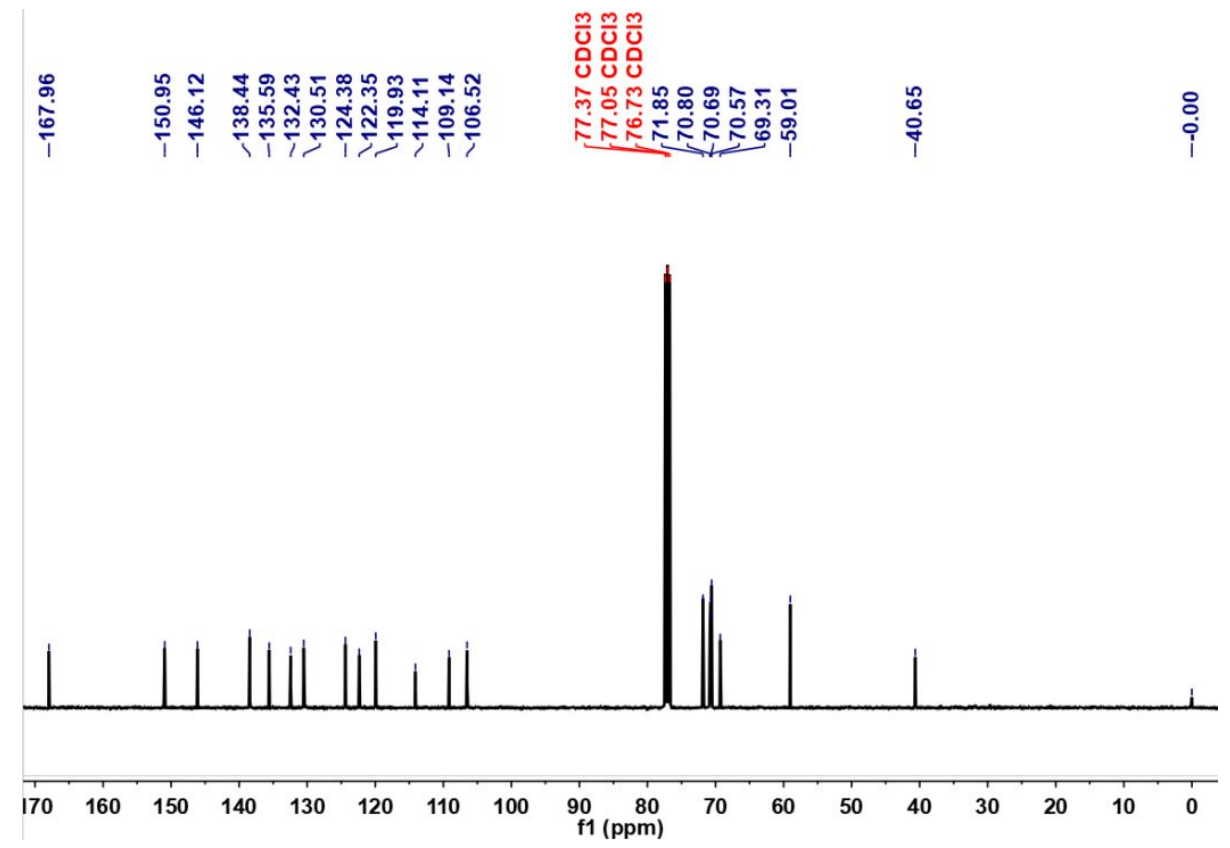

Figure S11. ${ }^{13} \mathrm{C}$ NMR spectrum of TEG-ID(TPCN)2 in $\mathrm{CDCl}_{3}$ with TMS as internal standard. 


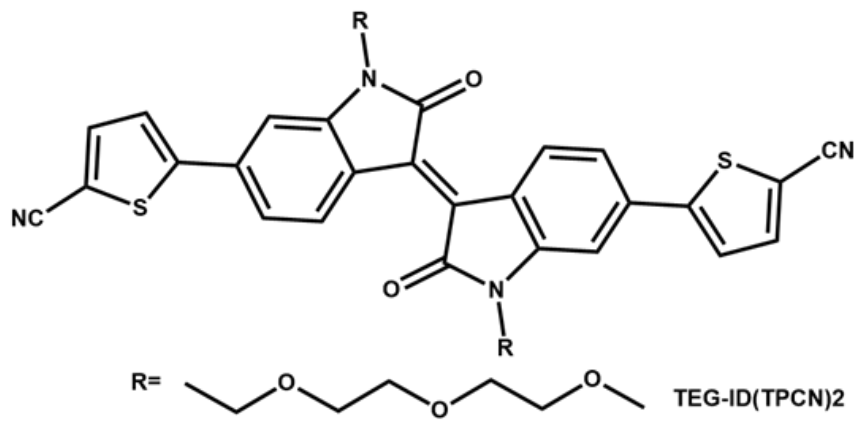

Theoretical Elemental Analysis: N:7.29 C:62.48 H:5.24

EagerSmart Summarize Results

Date : 6/19/2020 at 12:42:09

Method Name : CHNS

Method Filename : CHN test.mth

Element of

Nitrogen

Carbon

Hydrogen

7.49

62.36

5.20

Figure S12. Elemental analysis of TEG-ID(TPCN)2. 